\title{
Mammalian target of rapamycin overexpression antagonizes chronic hypoxia-triggered pulmonary arterial hypertension via the autophagic pathway
}

\author{
LINGXIA LI ${ }^{1}$, XIAOCHUANG WANG ${ }^{2}$, LINA WANG ${ }^{2}$, LI QU $^{2}$, XINYE ZHU ${ }^{2}$, MANXIANG LI ${ }^{3}$, \\ XIAOYAN DANG ${ }^{2}$, PING LI $^{2}$, YANXIA GAO ${ }^{2}$, ZHUO PENG $^{2}$, LONGFEI PAN ${ }^{2}$ and LI WAN ${ }^{2}$ \\ ${ }^{1}$ The Cadre Ward; Departments of ${ }^{2}$ Emergency Medicine and ${ }^{3}$ Respiratory Diseases, \\ The Second Affiliated Hospital of Medical College, Xi'an Jiaotong University, Xi'an, Shaanxi 710004, P.R. China
}

Received October 28, 2014; Accepted May 18, 2015

DOI: $10.3892 / \mathrm{ijmm} .2015 .2224$

\begin{abstract}
Pulmonary arterial hypertension (PAH) is a progressive pulmonary vascular disorder with high morbidity and mortality, and is characterized by excessive growth of endothelial cells. Recently, the mammalian target of rapamycin (mTOR) has attracted increasing attention due to its potential as a therapeutic target against certain diseases associated with proliferative and metabolic abnormalities. However, the effect on mTOR on PAH has not yet been elucidated. In the present study, a marked downregulation of mTOR was observed in PAH patients. Following construction of a mouse model of PAH by chronic exposure to hypoxia, adenovirus-mediated upregulation of mTOR significantly attenuated right ventricular systolic pressure, right ventricular hypertrophy and wall thickness of pulmonary arterioles, indicating a protective effect of mTOR on PAH. Further analysis confirmed that mTOR overexpression inhibited autophagy triggered by hypoxia through blocking light chain 3 II expression and increasing p62 levels. In vitro, hypoxia enhanced the proliferation of human pulmonary artery endothelial cells (PAECs), which was markedly abrogated by mTOR overexpression. Of note, upregulation of mTOR inhibited the hypoxia-induced autophagy pathway, which contributed to cell proliferation, while silencing of autophagy by RNA interference with ATG5 significantly inhibited cell prolifera-
\end{abstract}

Correspondence to: Dr Xiaochuang Wang, Department of Emergency Medicine, The Second Affiliated Hospital of Medical College, Xi'an Jiaotong University, 157 West 5th Road, Xi'an, Shaanxi 710004, P.R. China

E-mail: xiaochuang_wang@163.com

Abbreviations: PAH, pulmonary arterial hypertension; mTOR, mammalian target of rapamycin; RVSP, right ventricular systolic pressure; PAECs, pulmonary artery endothelial cell; PASMCs, pulmonary artery smooth muscle cells; GFP, green fluorescent protein; PVDF, polyvinylidene difluoride; BW, body weight; RVW, right ventricle weight; $L V$, left ventricle

Key words: pulmonary arterial hypertension, mammalian target of rapamycin, pulmonary artery endothelial cell, autophagy tion. In conclusion, the results of the present study suggested a potential protective effect of mTOR on the progression of PAH by suppressing PAEC proliferation through blocking the autophagic pathway. Therefore, the present study suggested that mTOR is a promising therapeutic agent against PAH.

\section{Introduction}

Pulmonary arterial hypertension (PAH) ranks as a fatal disease with high morbidity and mortality, and is clinically identified by detecting the mean pulmonary artery pressure (PAP) at $>25$ $\mathrm{mmHg}$ at rest or $>30 \mathrm{mmHg}$ with exercise. PAH is now widely accepted as a progressive disorder, which is characterized by a marked increase in pulmonary arterial pressure, vascular remodeling and right ventricular hypertrophy, leading to shortness of breath, dizziness, fainting, leg swelling and other symptoms (1-3). In spite of the number of studies available, the underlying mechanisms of the pathological progression of PAH have largely remained elusive.

Pulmonary hypertension often occurs either as a primary or secondary disease following cardiac or pulmonary diseases. Accumulating evidence has shown that the histological lung biopsies in PAH patients display critical vascular remodeling, including smooth muscle cell proliferation with medial hypertrophy and endothelial cell proliferation with subsequent intimal thickening (4,5). Endothelial cell proliferation and smooth muscle cell (SMC) migration lead to the formation of so-called plexiform lesions, which are characteristics of primary and secondary pulmonary hypertension $(1,6)$. The hyperplasia of pulmonary artery smooth muscle cells (PASMCs) has been demonstrated in monocrotaline (MCT)-induced PAH rats, in which pre-conditioning with fluoxetine significantly inhibited PASMC proliferation and subsequent PH development (7). In addition, abnormal endothelial cell growth in the lungs of $\mathrm{PH}$ patients and hypoxia-induced PAH models has been observed, which is therefore a characteristic of PAH $(6,8)$.

Autophagy is an evolutionarily conserved lysosomal degradation pathway and is involved in various vascular diseases, including atherosclerosis, heart failure and ischemia/re-perfusion injury (9-11). Recently, the pivotal roles of autophagy in pulmonary diseases, including acute lung injury, pulmonary 
hypertension and chronic obstructive pulmonary disease, have drawn increasing interest (12). It has been reported that apelin overexpression significantly inhibited PASMC proliferation and migration by suppressing the autophagic pathway, indicating a potential role of autophagy in PAH (13). Furthermore, impaired angiogenesis has been corroborated in fetal lambs with persistent $\mathrm{PH}$; however, blocking of autophagy enhanced angiogenesis, implying a possible beneficial function of autophagy in PAH progression (14). Numerous studies have demonstrated a critical role of mammalian target of rapamycin (mTOR) in regulating the autophagic pathway $(15,16)$. mTOR is known as a well-conserved serine/threonine kinase that exerts a pivotal function in the signaling network controlling cell metabolism, growth, proliferation and survival in response to various environmental factors. Recently, mTOR pathways were proved to be involved in the apelin-induced inhibitory effect of autophagy on PASMC proliferation (13). Even though numerous studies have validated the negative effect of mTOR on the autophagic pathway, its effect on PAH has remained elusive.

The present study examined the expression of mTOR in lung tissues from PAH patients. Furthermore, the function of mTOR in PAH triggered by chronic hypoxia was explored, and the underlying mechanism was investigated.

\section{Materials and methods}

Reagents. Rabbit anti-human mTOR monoclonal antibody (\#2972) was obtained from Cell Signaling Technology, Inc., (Danvers, MA, USA). Rabbit anti-mouse mTOR polyclonal antibody (ab2732) was purchased from Abcam (Cambridge, MA, USA). The polyclonal antibody against mouse light chain (LC)3 (NB100-2220) was from NOVUS (Littleton, CO, USA). Rabbit polyclonal anti-human LC3 (ab128025), p62 (ab91526) and -autophagy-related 5 (ATG5; ab78073) were from Abcam. Rabbit anti-human p62 antibodies (sc-25730) were from Santa Cruz Biotechnology (Dallas, TX, USA).

Specimen collections. The present study comprised 13 patients (aged 28-60 years) with PAH and six control subjects (aged 27-62 years). All human lung tissues were from volunteers with PAH and were processed according to the recommendations of the Second Affiliated Hospital of Medical College, Xi'an Jiaotong University (Xi'an, China) (17). Normal lungs were obtained from 6 patients who died from traumatic injury unrelated to the lung. The study was conducted in compliance with the Helsinki Declaration and all patients gave written informed consent. All specimens were preserved in liquid nitrogen for subsequent experiments.

Construction of the recombinant adenoviral vector. The mouse mTOR cDNA fragments were subcloned into the $\mathrm{p}$ adenovirus (Ad) Track-cytomegalovirus (CMV) vector expressing green fluorescent protein (GFP) to construct the recombinant vector pAdTrack-CMV-mTOR-GFP.

The recombinant shuttle plasmids pAdTrack-CMV and pAdEasy-1 were then homogeneously re-combined in the Escherichia coli strain BJ5183 (Stratagene, La Jolla, CA, USA). The obtained recombinant plasmids were then transfected into 293 cells (ATCC, Rockville, MD, USA) to produce the recombinant adenovirus. Following amplification and purification, the p24 ELISA kit (Cell Biolabs, Inc., San Diego, CA, USA) was used to determine the virus titers and vectors were then stored at $-80^{\circ} \mathrm{C}$ until use.

Animal model of pulmonary hypertension. For experiments requiring animal use, thirty wild-type C57BL/6 mice (nine weeks old; male; weighing 20-25 g) were used. All animal experiments were performed with the approval from the Institutional Animal Care and Use Committee of the Second Affiliated Hospital of Medical College, Xi'an Jiaotong University (Xi'an, China). All animals were housed under a controlled 12-h light/ dark cycle and temperature conditions, with free access to water and chow. The mice were injected with the virus via their tail veins. For each injection, $\sim 0.2-\mathrm{ml}$ viral suspension with a titer of $1 \times 10^{7} \mathrm{IU} / \mathrm{ml}$ was administered every other day. Mice were then exposed to hypoxia $\left(10 \% \mathrm{O}_{2}\right)$ or normoxia in a chamber for three weeks. The mice were then anaesthetized with sodium pentobarbital $(50 \mathrm{mg} / \mathrm{kg}$ ) (Beyotime Biotech, Shanghai, China) for lung tissue harvesting. Western blot analysis was performed to determine mTOR levels and autophagy.

Hemodynamic studies and right ventricular hypertrophy. Three weeks following injection with the vector, all animals were anesthetized with sodium pentobarbital $(60 \mathrm{mg} / \mathrm{kg})$ for hemodynamic assessment. The body weight (BW) of mice was recorded, and a $23 \mathrm{G}$ needle was then inserted into the right ventricle for measuring the right ventricle (RV) pressure. For RV hypertrophy analysis, the heart was removed. The ratio of right ventricle weight to body weight (RVW/BW) or by the ratio of RVW to combined left ventricle and septum weight $[\mathrm{RV} /(\mathrm{LV}+\mathrm{Sep})]$ was detected to assess the degree of RV hypertrophy. In addition, the wall thickness in all groups of animals was also measured and calculated as: Wall thickness $(\%)=$ $\left(\right.$ area $_{\text {ext }}-$ area $\left._{\text {int }}\right) /$ area $_{\text {ext }} \times 100$. The area $\mathrm{ext}_{\text {represents the external }}$

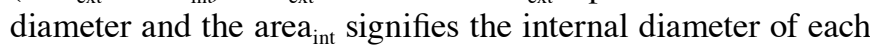
vessel. All parameters were analyzed using PVAN 3.5 software (Millar Instruments, Houston, TX, USA).

Cell culture. Human pulmonary artery endothelial cells (PAECs) were purchased from Lonza (Basel, Switzerland). Passages from PAEC 5-8 were grown to $\sim 80 \%$ confluence in endothelial cell growth medium-2 (EGM-2; Lonza Walkersville Inc., Walkersville, MD, USA) containing EGM-2 SingleQuots ${ }^{\mathrm{TM}}$ (Lonza) and 2\% fetal bovine serum (FBS; (Lonza). Cells were then transfected with $2 \mathrm{nmol} / \mathrm{l}$ Ad-GFP or Ad-mTOR vector using the DharmaFECT transfection reagent (Dharmacon, Lafayette, CO, USA), followed by exposure to hypoxia $\left(10 \% \mathrm{O}_{2}\right)$ or normoxia for $72 \mathrm{~h}$. All cells were maintained at $37^{\circ} \mathrm{C}$.

Small interfering (si)RNA transfection. For specifically silencing ATG5 expression, the targeted siRNA fragments of ATG5 were designed and obtained from Qiagen (Hilden, Germany) (NM_004849). The scrambled siRNA (NC) was obtained form Santa Cruz Biotechnology. For transfection, cells were seeded into 24-well micro-plates to reach $40-50 \%$ confluence with a density of $1 \times 10^{5}$ cells/well. Subsequently, $2 \mu \mathrm{g} / \mathrm{ml}$ ATG5 siRNA or scrambled siRNA were transfected into cells together with $1 \mathrm{ml}$ Lipofectamine ${ }^{\mathrm{TM}}$ RNAi-MAX (Invitrogen, Carlsbad, CA, USA) using the GeneSilencer ${ }^{\circledR}$ siRNA transfection reagent (Gene Therapy Systems, Inc., San 
Diego, CA, USA). Twenty-four hours later, the transfection efficiency was assessed by western blot analysis.

Reverse transcription quantitative polymerase chain reaction $(R T-P C R)$. Total RNA from the above tissue samples was extracted with TRIzol reagent (Sigma-Aldrich, St Louis, MO, USA). Subsequently, RT was performed using ImProm II reverse-transcriptase (Promega, Madison, WI, USA) with oligo-dT priming to obtain the cDNA. To evaluate the mRNA levels of mTOR in tissues, the obtained cDNA was used as a template to perform PCR amplification with the SYBR Premix Ex Taq ${ }^{\mathrm{TM}}$ II kit (Takara, Shiga, Japan) using an ABI PRISM Sequence Detector System 7500 (Applied Biosystems, Foster City, CA, USA). The specific primers for mTOR were 5'-ATTTGATCAGGTGTGCCAGT-3' (forward sequence) and 5'-GCTTAGGACATGGTTCATGG-3' (reverse sequence). Each $20-\mu 1$ reaction system was comprised of $2 \mu \mathrm{l}$ cDNA, $10 \mu 1 \mathrm{SYBR}^{\circledR}$ Premix EX Taq ${ }^{\mathrm{TM}}$ II and $10 \mu \mathrm{mol} / 1$ of forward and reverse primers. The PCR program was $95^{\circ} \mathrm{C}$ for $10 \mathrm{~min}$, followed by 38 PCR cycles $\left(95^{\circ} \mathrm{C}\right.$ for $10 \mathrm{sec}, 56^{\circ} \mathrm{C}$ for $40 \mathrm{sec}$, $72^{\circ} \mathrm{C}$ for $30 \mathrm{sec}$ ) and a final extension for $5 \mathrm{~min}$ at $72^{\circ} \mathrm{C}$, followed by a standard melting curve analysis. Amplification reactions were performed in triplicate for each sample and the results were normalized to $\beta$-actin (11). All primers were obtained from GenePharma (Shanghai, China). The $2^{-\Delta \Delta C}$ method was used to quantify results (11).

Western blot analysis. Following lysis with radioimmunoprecipitation assay lysis buffer $(100 \mathrm{mM} \mathrm{NaCl}, 50 \mathrm{mM}$ Tris-HCl pH 7.5, $1 \%$ Triton X-100, 1 mM EDTA, $10 \mathrm{mM} \beta$-glycerophosphate, $2 \mathrm{mM}$ sodium vanadate and protease inhibitor; Beyotime Biotech), the protein concentration in the above samples was measured using the Pierce ${ }^{\mathrm{TM}}$ BCA Protein Assay Kit (23227; Pierce, Rockford, IL, USA). Approximately $100 \mu 1$ protein (25 $\mu \mathrm{g} /$ lane) was subjected to $12 \%$ SDS-PAGE, followed by the transfer onto a polyvinylidene difluoride membrane (Millipore, Bedford, MA, USA) in a semi-dry trans-blot apparatus. Then, $5 \%$ non-fat dry milk in phosphate-buffered saline was added to block the non-specific binding at $4^{\circ} \mathrm{C}$ overnight. The membranes were then incubated with primary antibodies against mTOR (1:1,000), light chain LC3 I, LC3 II (1:500), p62 (1:200) and ATG5 (1:500) for $1 \mathrm{~h}$ at room temperature. Following three washes with Tris-buffered saline containing Tween 20, horseradish peroxidase (HRP)-conjugated secondary antibodies were introduced for $1 \mathrm{~h}$. LumiGLo reagent (Pierce) was added to detect the bound antibodies. The images were obtained on Kodak film and quantified using ImageJ software version 1.46 (National Institutes of Health, Bethesda, MD, USA).

Cell proliferation assay. To assess cell proliferation, an MTT assay was performed. Briefly, cells were seeded into 96-well plates at a density of $1 \times 10^{5}$ cells/well. Following pre-conditioning with Ad-mTOR transfection, the culture medium was replaced with fresh medium containing $500 \mu \mathrm{g} / \mathrm{ml}$ MTT reagent. Following 5 hours of incubation at $37^{\circ} \mathrm{C}, 200 \mu \mathrm{l}$ isopropanol was added to dissolve the formazan product. Cell viability was then analyzed by detecting the absorbance of MTT at $590 \mathrm{~nm}$ with a micro-ELISA reader (3550; Bio-Rad Laboratories, Inc., Hercules, CA, USA). All samples were performed in triplicate.
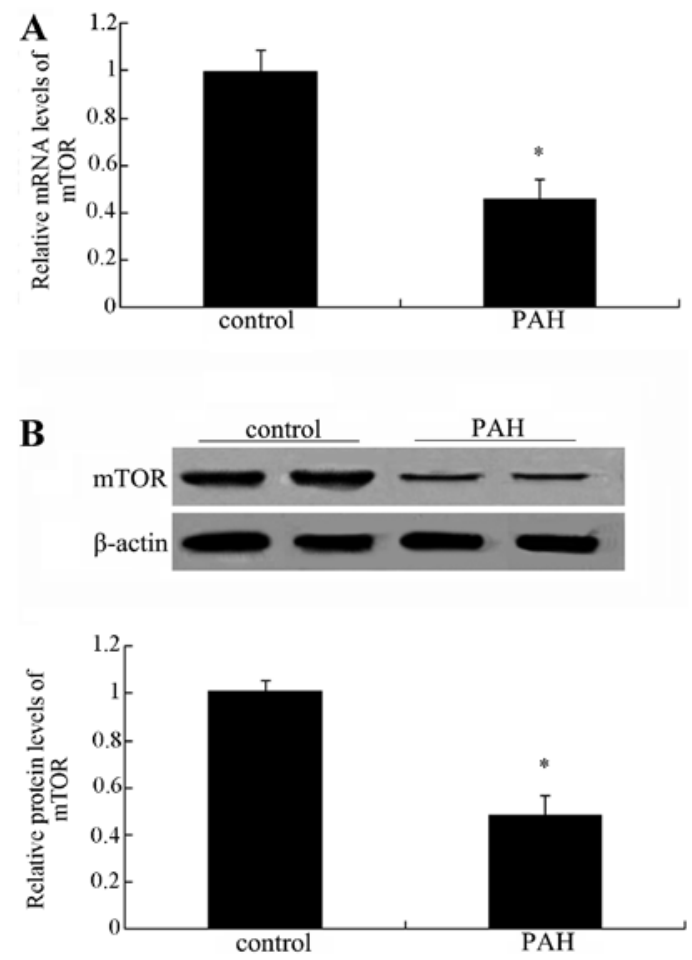

Figure 1. mTOR was decreased in lung tissues from PAH patients. (A) mRNA levels of mTOR in 13 patients (aged 28-60 years) with PAH and six control subjects (aged 27-62 years) were analyzed by reverse transcription polymerase chain reaction. (B) The corresponding protein levels were detected by western blot analysis. Values are expressed as the mean \pm standard deviation $(\mathrm{n}=3)$. ${ }^{*} \mathrm{P}<0.05$ vs. control groups. mTOR, mammalian target of rapamycin; PAH, pulmonary arterial hypertension.

Statistical analysis. All assays were performed in triplicate and values are expressed as the mean \pm standard deviation. SPSS 13.0 (SPSS, Inc., Chicago, IL, USA) was used for statistical analysis. Student's t-test was performed using GraphPad InStat Statistics software (version 1.12; GraphPad, Inc., La Jolla, CA, USA) to determine statistical significance. $P<0.05$ was considered to indicate a statistically significant difference between values.

\section{Results}

mTOR is downregulated in patients with PAH. To clarify the association between mTOR and PAH, lung tissues from normal patients and patients with $\mathrm{PAH}$ were collected and subjected to PCR and western blot analysis of mTOR. As shown in Fig. 1A, a significant downregulation of mTOR mRNA levels was observed compared with those in the normal groups $(\mathrm{P}<0.05)$. Simultaneously, the protein levels of mTOR were also markedly decreased in patients with PAH (Fig. 1B). Collectively, these results suggested a marked downregulation of mTOR in patients with PAH, indicating an association between mTOR and the development of PAH.

ElevatedmTORexpressionattenuateschronic hypoxia-induced pulmonary artery hypertension. Based on the above results, the present study evaluated the possible function of mTOR in the pathological progression of PAH in vivo. Following injection of recombinant Ad-mTOR, elevated mTOR protein levels 
A
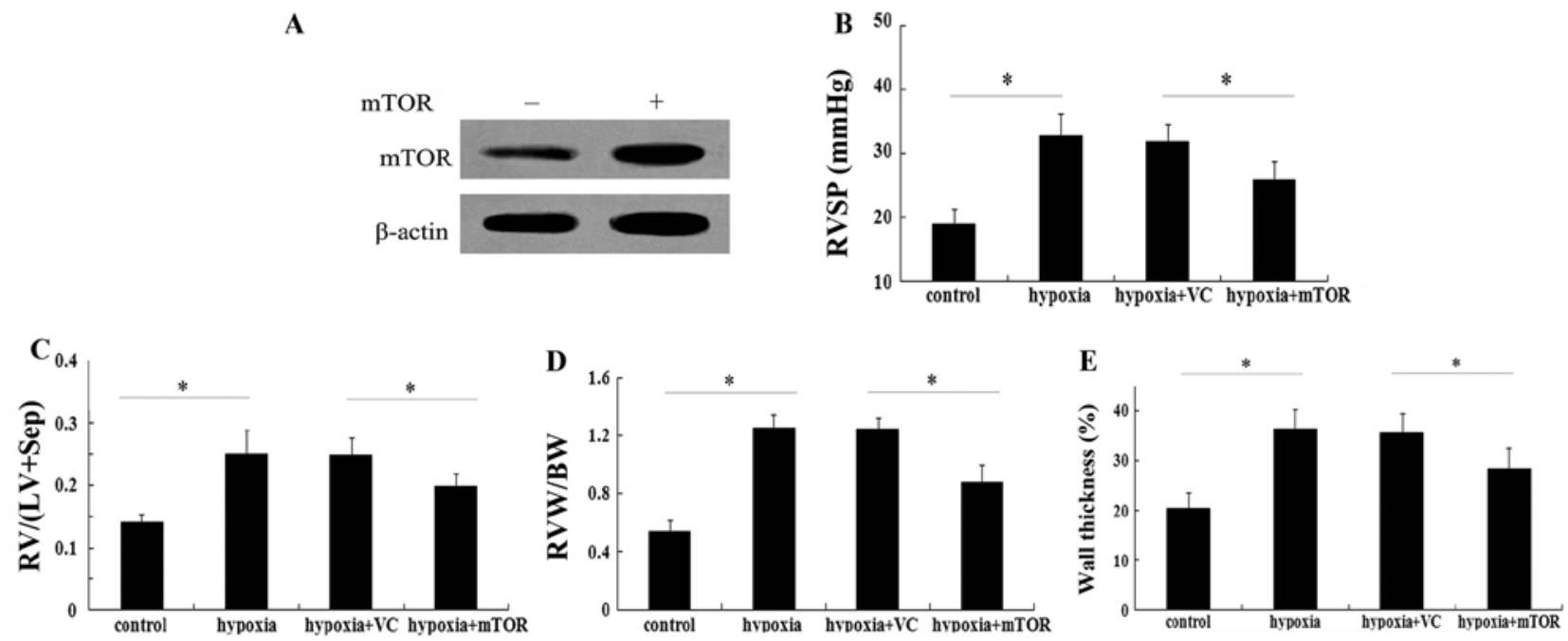

Figure 2. Administration of mTOR inhibits chronic hypoxia-induced pulmonary artery hypertension. Following injection of recombinant Ad-mTOR into wild-type C57BL/6 mice (nine weeks old), the mice were exposed to hypoxia $\left(10 \% \mathrm{O}_{2}\right)$ or normoxia for three weeks. (A) The expression levels of mTOR were assessed by western blot analysis. (B) The effect of mTOR overexpression on RVSP was also assessed. The ratios of (C) RV/(LV + Sep) and (D) RVW/BW were measured to analyze right ventricular hypertrophy. (E) The corresponding effect on wall thickness was also determined. Values are expressed as the mean \pm standard deviation $(\mathrm{n}=3)$. "P<0.05. RVSP, right ventricular systolic pressure; Ad, adenovirus; RVW, right ventricle weight; BW, body weight; LV, left ventricle; Sep, septum weight; mTOR, mammalian target of rapamycin; VC, vector control.
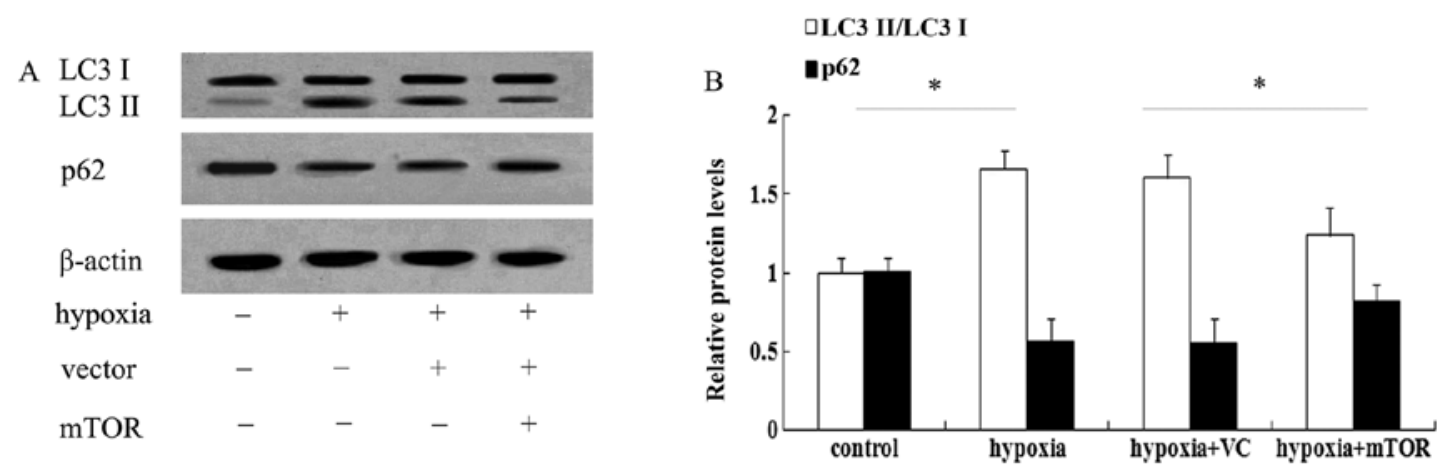

Figure 3. Increased mTOR expression abrogates the increased autophagy in mice with hypoxia-induced pulmonary arterial hypertension. (A) Following lysis of the collected lung tissues, the protein levels of autophagy markers (LC3 II/LC3 I and p62) were evaluated by western blot analysis. (B) Quantitative densitometric analysis of western blots was performed using Image J software. Values are expressed as the mean \pm standard deviation ( $\mathrm{n}=3$ ). $\mathrm{P}<0.05$. LC, light chain; mTOR, mammalian target of rapamycin; VC, vector control.

were detected in C57BL/6 mice with chronic hypoxia-induced pulmonary artery hypertension (Fig. 2A). Of note, mTOR overexpression vector administered for three weeks markedly inhibited the elevation of right ventricular systolic pressure (RVSP) in mice exposed to hypoxia ( $\mathrm{P}<0.05$; Fig. 2B). In terms of right ventricular hypertrophy, hypoxia increased the ratio of $\mathrm{RV} /(\mathrm{LV}+\mathrm{Sep})$ by $\sim 1.8$-fold relative to the baseline value, which was markedly attenuated in the group injected with Ad-mTOR $(\mathrm{P}<0.05$; Fig. 2C). Consistent with the above results, the increase in the ratio of RVW/BW induced by hypoxia was significantly reduced in the mTOR overexpression group $(\mathrm{P}<0.05$; Fig. 2D). Of note, exposure to hypoxia exaggerated vascular remodeling with an obvious increase in wall thickness of pulmonary arterioles $(36.25 \pm 4.10 \%$, compared with $20.51 \pm 3.11 \%$ in the control group; $\mathrm{P}<0.05$ ) (Fig. 2E). When mTOR was overexpressed, this upregulation in wall thickness of pulmonary arterioles was markedly mitigated $(\mathrm{P}<0.05)$.
These results suggested a potential protective effect of mTOR against PAH.

mTOR overexpression abrogates increased autophagy in mice with PAH. It is widely accepted that mTOR is associated with autophagy. To test whether the underlying mechanism of the beneficial effect of mTOR in PAH involves autophagic pathways, LC3 was assessed, which is a known marker for autophagy (17). As shown in Fig. 3A, exposure to hypoxia for three weeks enhanced the expression of LC3 II and induced a 1.56-fold increase in the ratio of LC3 II/LC3 I in the lungs of mice with PAH (Fig. 3B). However, mTOR overexpression significantly attenuated the increase in LC3 levels $(\mathrm{P}<0.05)$. As a common autophagic substrate, p62 serves as a useful marker for measuring autophagic flux $(11,14)$. Following exposure to hypoxia, the expression levels of p62 were obviously abrogated in mice with PAH (Fig. 3), which was restored in the 
A
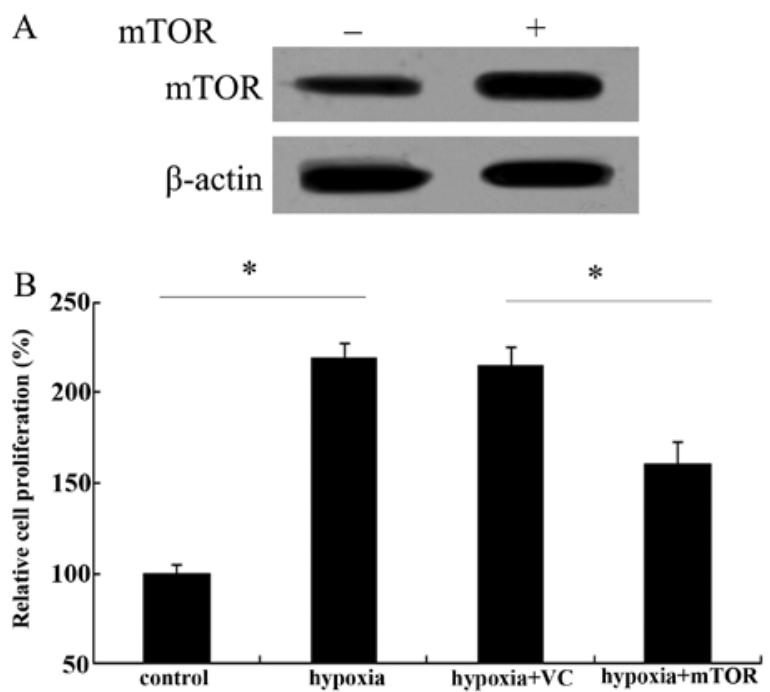

Figure 4. Upregulation of mTOR reduces hypoxic proliferation of PAECs. (A) After transfection with Ad-mTOR, the expression levels of mTOR in PAECs were assessed by western blotting. (B) The effect of mTOR transfection on hypoxia-triggered cell proliferation was analyzed by MTT assay. Values are expressed as the mean \pm standard deviation $(n=3)$. ${ }^{*} \mathrm{P}<0.05$. PAECs, pulmonary artery endothelial cells; Ad, adenovirus; mTOR, mammalian target of rapamycin.

mTOR-overexpressing group. The above results suggested that mTOR may exert a protective effect against PAH by inhibiting the autophagic pathway.

mTOR overexpression impedes hypoxic proliferation of PAECs. It is well known that abnormal endothelial cell growth occurs in pulmonary arteries and lungs of patients with $\mathrm{PAH}(8)$. Therefore, the effect of mTOR on PAEC proliferation was assessed. After transfected with Ad-mTOR, the expression levels of mTOR protein were markedly upregulated in PAECs (Fig. 4A). Following exposure to chronic hypoxia, an $\sim 2.17$-fold increase in cell proliferation ability was demonstrated (Fig. 4B). However, mTOR overexpression obviously attenuated this increase in cell proliferation triggered by hypoxia $(\mathrm{P}<0.05)$, indicating a critical role of mTOR in the hypoxic proliferation of PAECs.

mTOR inhibits hypoxia-induced cell autophagy in PAECs. As the effect of mTOR on hypoxia-induced PAEC proliferation had been confirmed, it was next sought to elucidate whether mTOR inhibited autophagy in PAECs. Western blot analysis showed that hypoxia induced an obvious increase in the LC3 II/LC3 I ratio, concomitant with the marked upregulation of ATG5-12 levels (Fig. 5A). Quantification of the blots revealed that hypoxia triggered $\sim 1.87$ - and 1.91-fold increases in LC3 and ATG5-12 levels, respectively $(\mathrm{P}<0.05)$, which were abrogated by mTOR overexpression $(\mathrm{P}<0.05)($ Fig. $5 \mathrm{~B})$. Furthermore, an obvious 0.48 -fold downregulation of p62 levels was noted in PAECs exposed to hypoxia $(\mathrm{P}<0.05)$, which was partly restored by mTOR overexpression $(\mathrm{P}<0.05)$ (Fig. 5C).

Blocking of autophagy antagonizes cell proliferation of PAECs. To explore the effect of autophagy on the proliferation of PAECs, ATG5 siRNA was used to silence the expression of ATG5-12. As shown in Fig. 6A, ATG5 siRNA transfection markedly abrogated the protein expression of ATG5-12 in

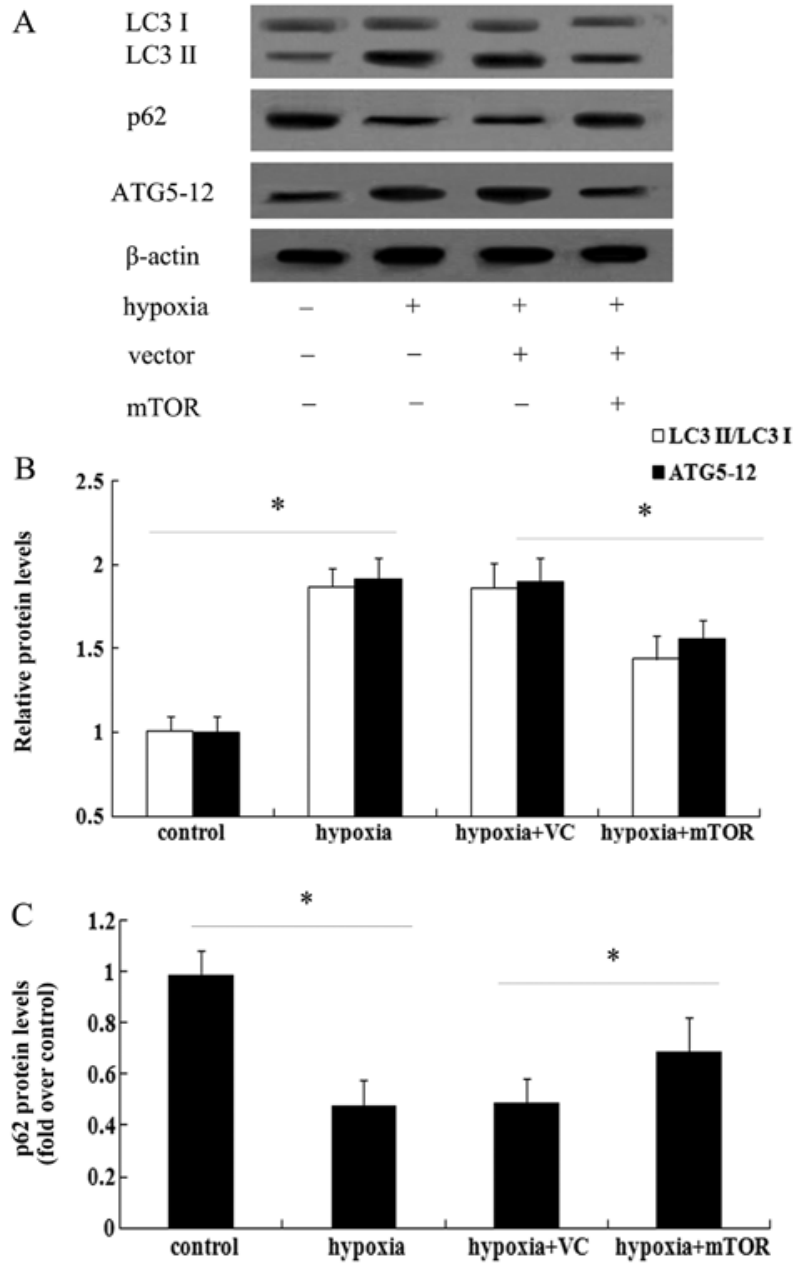

Figure 5. Effect of mTOR on hypoxia-induced cell autophagy in PAECs. Human PAECs were pre-conditioned with $2 \mathrm{nmol} / \mathrm{l}$ Ad-green fluorescence protein or Ad-mTOR and subsequently exposed to hypoxia $\left(10 \% \mathrm{O}_{2}\right)$ or normoxia for $72 \mathrm{~h}$. (A) The expression levels of autophagy markers (LC3 II/LC3 I, ATG5-12 and p62) were determined by western blot analysis. Quantified protein levels of (B) LC3 II/LC3 I and ATG5-12 and (C) p62. Values are expressed as the mean \pm standard deviation $(n=3)$. ${ }^{*} \mathrm{P}<0.05$. LC, light chain; mTOR, mammalian target of rapamycin; VC, vector control; PAECs, pulmonary artery endothelial cells; Ad, adenovirus; LC, light chain; ATG5, autophagy-related 5.

PAECs. Of note, ATG5 silencing reduced hypoxia-induced increases in LC3 II levels (Fig. 6B). Consistently, a marked upregulation of p62 protein levels was also observed when blocking ATG5 levels, indicating that ATG5 knockdown inhibited the cell autophagy pathway. Furthermore, silencing of ATG5 significantly reduced hypoxia-induced cell proliferation $(\mathrm{P}<0.05)$ (Fig. 6C).

\section{Discussion}

Pulmonary hypertension $(\mathrm{PH})$ is a progressive pulmonary vascular disorder comprising increased proliferation ability of PASMCs and PAECs, resulting in high morbidity and mortality $(8,18,19)$. As a well-conserved serine/threonine kinase, mTOR controls various major cellular processes and is increasingly being associated with pathological conditions (20). Recently, mTOR has attracted broad interest of scientists and clinicians due to being a potential therapeutic target against a number of diseases associated with prolif- 
A

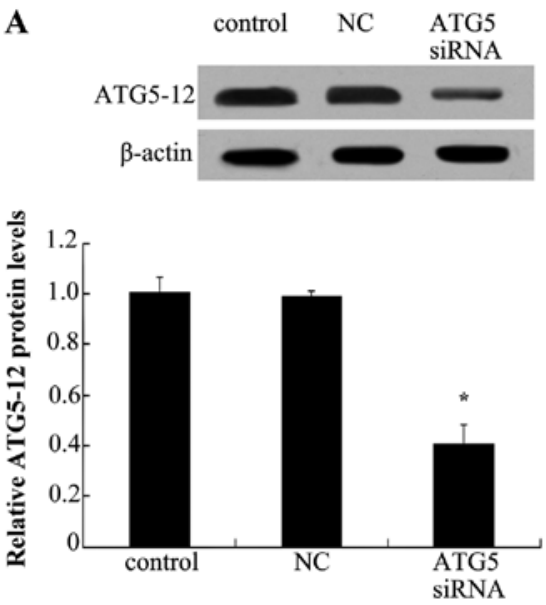

B
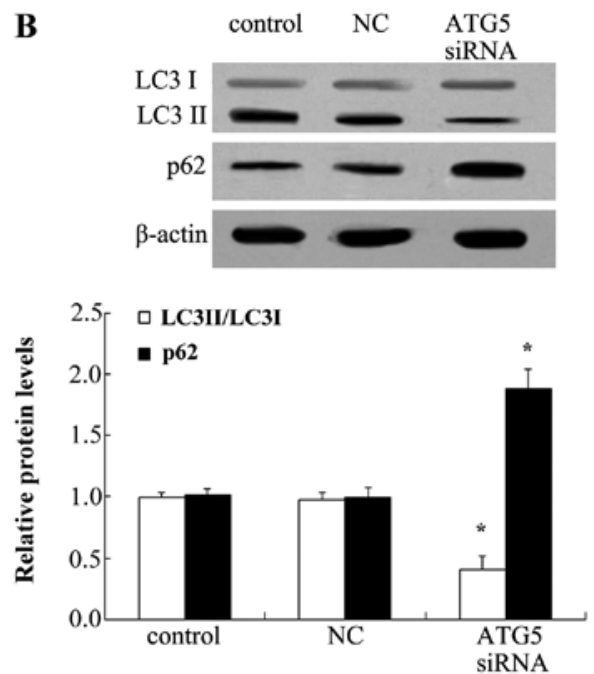

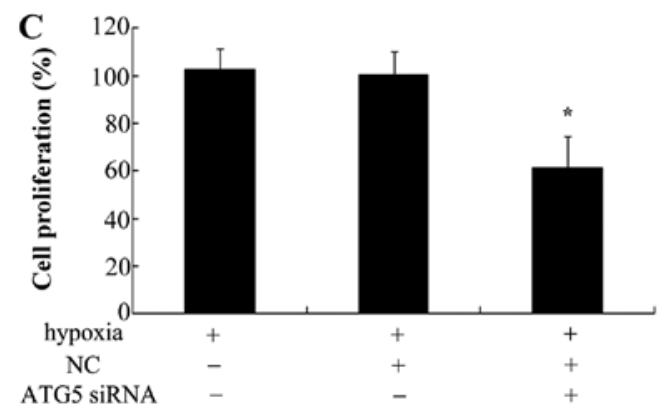

Figure 6. Blocking of autophagy inhibited cell proliferation of PAECs. To investigate the role of autophagy in hypoxia-induced cell proliferation, ATG5 siRNA was transfected into PAECs, followed by exposure of the cells to hypoxia. (A) The silencing effect of ATG5-12 expression was analyzed by western blotting. (B) The effect of ATG5 silencing on autophagy markers (LC3 II/LC3 I and p62) was explored. (C) Furthermore, the effect of AGT5-12 silencing on cell proliferation was evaluated using an MTT assay. Values are expressed as the mean \pm standard deviation $(\mathrm{n}=3)$. ${ }^{*} \mathrm{P}<0.05$ vs. groups exposed to hypoxia. NC, negative control; siRNA, small interfering RNA, PAECs, pulmonary artery endothelial cells; LC, light chain; ATG5, autophagy-related 5

erative and metabolic abnormalities, including atherosclerosis, cancer and neurodegeneration $(21,22)$. However, to date, its function in PAH has remained elusive. The present study reported an obvious downregulation of mTOR in lung tissues from patients with $\mathrm{PAH}$, indicating a critical role of mTOR in the development of PAH.

Pulmonary hypertension has been proven to contribute to the morbidity and mortality of adult and pediatric patients with various lung and heart diseases, which are mainly associated with persistent or intermittent hypoxia (23). The fact that hypoxia can induce PAH and significant structural remodeling of pulmonary arteries (PAs) in experimental models has been widely confirmed and will contribute to disease development $(24,25)$. To investigate the role of mTOR in PAH, a mouse model of PAH was established in the present study by exposing the animals to chronic hypoxia. Through simultaneous injection with a specific adenovector, mTOR-overexpressing mice with PAH were successfully generated. Following prolonged exposure to hypoxia, a significant enhancement of the RVSP was observed, which was obviously attenuated in C57BL/6 mice injected with Ad-mTOR. Furthermore, mTOR overexpression markedly abrogated right ventricular hypertrophy as decreased hypoxia-induced increases in the $\mathrm{RV} /(\mathrm{LV}+\mathrm{Sep})$ and RVW/BW ratios. Of note, the wall thickness of pulmonary arterioles was markedly mitigated by overexpression of mTOR. Therefore, the above results confirmed the critical protective role of mTOR in the progression of PAH.
Autophagy is a lysosomal pathway which can degrade intracellular organelles and proteins to maintain cellular homeostasis (26). The microtubule-associated protein LC3 is frequently used as a specific marker to monitor macroautophagy in vitro and in vivo, and increases in LC3 II indicate the induction of autophagosome formation (27). The present study confirmed that autophagy was elevated in PAH, which was previously observed in monocrotaline-triggered PAH in rats (28). A recent study confirmed that inhibition of autophagy was able to prevent pulmonary hypertension in rats (29). The fact that mTOR is known as a negative regulator of autophagy has been widely observed (30). To further clarify the underlying mechanism of the protective effect of mTOR against PAH, the effect of mTOR on the autophagic pathway was investigated. As expected, overexpression of mTOR abrogated the expression levels of LC3 II induced by chronic hypoxia. As a common autophagic substrate, p62 is recognized as a useful marker for measuring autophagic flux $(11,14)$. In the present study, mTOR overexpression markedly ameliorated the inhibitory effect of hypoxia on p62 expression levels. These results indicated that autophagic pathways are involved in the mTOR-mediated attenuation of PAH induced by chronic hypoxia.

It has been demonstrated that hypoxia can contribute to the development and progression of PAH, mainly by de-regulating endothelial cell functions, including excessive growth of endothelial cells (31). Abnormal endothelial cell growth in the lungs and pulmonary arteries of $\mathrm{PH}$ patients has been reported 
previously $(8,32)$. In the present study, hypoxia treatment markedly enhanced the proliferation of endothelial cells. Although a previous study has suggested that hypoxia stimulation failed to induce pulmonary artery endothelial cell proliferation (33), other studies have reported results similar to those of the present study $(34,35)$. The present study manifested that overexpression of mTOR significantly reduced hypoxia-induced PAEC proliferation. Of note, stimulation with hypoxia also enhanced the autophagic pathway as evidenced by the upregulation of LC3 II and ATG5-12, as well as the decease of p62 expression. ATG5 is an E3 ubiquitin ligase and is required for autophagy due to its role in autophagosome elongation (36). Following ATG5 knockdown with its specific siRNA, the autophagic pathway was obviously blocked. Of note, ATG5 silencing markedly inhibited hypoxia-induced PAEC proliferation, implying a critical role of mTOR in hypoxia-induced PAEC proliferation by regulating the autophagic pathway.

In conclusion, the present study reported an obvious downregulation of mTOR in PAH induced by chronic hypoxia. In the present study, high expression levels of mTOR were able to abrogate the development of hypoxia-triggered PAH by suppressing PAEC proliferation and through blocking the autophagic pathway. Accordingly, the present study illustrated the potential protective effect of mTOR against the development and progression of PAH and suggested mTOR as a promising therapeutic agent for the future development of anti-PAH therapies.

\section{Acknowledgements}

The present study was supported by the Key Project of the National Natural Science Foundation of China (no. 81330002) and the Social Development Technology Project of Shaanxi Province (no. 2015S099).

\section{References}

1. Tuder RM, Abman SH, Braun T, et al: Development and pathology of pulmonary hypertension. J Am Coll Cardiol 54: S3-S9, 2009.

2. McLaughlin VV, Davis M and Cornwell W: Pulmonary arterial hypertension. Curr Probl Cardiol 36: 461-517, 2011.

3. Voelkel NF, Gomez-Arroyo J, Abbate A, Bogaard HJ and Nicolls MR: Pathobiology of pulmonary arterial hypertension and right ventricular failure. Eur Respir J 40: 1555-1565, 2012.

4. Tuder RM, Marecki JC, Richter A, Fijalkowska I and Flores S: Pathology of pulmonary hypertension. Clin Chest Med 28: 23-42, 2007.

5. Rabinovitch M: Molecular pathogenesis of pulmonary arterial hypertension. J Clin Invest 118: 2372-2379, 2008.

6. Xu W and Erzurum SC: Endothelial cell energy metabolism, proliferation, and apoptosis in pulmonary hypertension. Compr Physiol 1: 357-372, 2011.

7. Guignabert C, Raffestin B, Benferhat R, et al: Serotonin transporter inhibition prevents and reverses monocrotaline-induced pulmonary hypertension in rats. Circulation 111: 2812-2819, 2005.

8. Masri FA, Xu W, Comhair SA, et al: Hyperproliferative apoptosis-resistant endothelial cells in idiopathic pulmonary arterial hypertension. Am J Physiol Lung Cell Mol Physiol 293 L548-L554, 2007.

9. Choi AM, Ryter SW and Levine B: Autophagy in human health and disease. N Engl J Med 368: 651-662, 2013.

10. Slomovitz BM and Coleman RL: The PI3K/AKT/mTOR pathway as a therapeutic target in endometrial cancer. Clin Cancer Res 18 $5856-5864,2012$.

11. Wang X, Li L, Niu X, et al: mTOR enhances foam cell formation by suppressing the autophagy pathway. DNA Cell Biol 33: 198-204, 2014.

12. Ryter SW, Nakahira K, Haspel JA and Choi AM: Autophagy in pulmonary diseases. Annu Rev Physiol 74: 377-401, 2012.
13. Zhang H, Gong Y, Wang Z, et al: Apelin inhibits the proliferation and migration of rat PASMCs via the activation of $\mathrm{PI} 3 \mathrm{~K} / \mathrm{Akt} / \mathrm{mTOR}$ signal and the inhibition of autophagy under hypoxia. J Cell Mol Med 18: 542-553, 2014.

14. Mizumura K, Cloonan SM, Haspel JA and Choi AM: The emerging importance of autophagy in pulmonary diseases. Chest 142: 1289-1299, 2012.

15. Nyfeler B, Bergman P, Wilson CJ and Murphy LO: Quantitative visualization of autophagy induction by mTOR inhibitors. Methods Mol Biol 821: 239-250, 2012.

16. Jung $\mathrm{CH}$, Ro SH, Cao J, Otto NM and Kim DH: mTOR regulation of autophagy. FEBS Lett 584: 1287-1295, 2010.

17. Zhang X, Li W, Hou Y, Niu Z, Zhong Y, Zhang Y and Yang S: Comparative membrane proteomic analysis between lung adenocarcinoma and normal tissue by iTRAQ labeling mass spectrometry. Am J Transl Res 6: 267-280, 2014.

18. Veyssier-Belot $\mathrm{C}$ and Cacoub P: Role of endothelial and smooth muscle cells in the physiopathology and treatment management of pulmonary hypertension. Cardiovasc Res 44: 274-282, 1999.

19. Perros F, Dorfmüller P, Souza R, et al: Fractalkine-induced smooth muscle cell proliferation in pulmonary hypertension. Eur Respir J 29: 937-943, 2007.

20. Laplante M and Sabatini DM: mTOR signaling in growth control and disease. Cell 149: 274-293, 2012.

21. Wang X, Li L, Li M, et al: Knockdown of mTOR by lentivirus-mediated RNA interference suppresses atherosclerosis and stabilizes plaques via a decrease of macrophages by autophagy in apolipoprotein E-deficient mice. Int J Mol Med 32: 1215-1221, 2013.

22. Zoncu R, Efeyan A and Sabatini DM: mTOR: from growth signal integration to cancer, diabetes and ageing. Nat Rev Mol Cell Biol 12: 21-35, 2010.

23. Stenmark KR, Fagan KA and Frid MG: Hypoxia-induced pulmonary vascular remodeling cellular and molecular mechanisms. Circ Res 99: 675-691, 2006

24. Orr R, Smith LJ and Cuttica MJ: Pulmonary hypertension in advanced chronic obstructive pulmonary disease. Curr Opin Pulm Med 18: 138-143, 2012.

25. Hoshikawa Y, Ono S, Suzuki S, et al: Generation of oxidative stress contributes to the development of pulmonary hypertension induced by hypoxia. J Appl Physiol 90: 1299-1306, 2001.

26. Mizushima N: Autophagy: process and function. Genes Dev 21: 2861-2873, 2007.

27. Kuma A, Matsui M and Mizushima N: LC3, an autophagosome marker, can be incorporated into protein aggregates independent of autophagy. Autophagy 3: 323-328, 2007.

28. Gomez-Arroyo JG, Farkas L, Alhussaini AA, et al: The monocrotaline model of pulmonary hypertension in perspective. Am J Physiol Lung Cell Mol Physiol 302: L363-L369, 2012.

29. Long L, Yang X, Southwood M, et al: Chloroquine prevents progression of experimental pulmonary hypertension via inhibition of autophagy and lysosomal bone morphogenetic protein type II receptor degradation. Circ Res 112: 1159-1170, 2013

30. Ravikumar B, Vacher C, Berger Z, et al: Inhibition of mTOR induces autophagy and reduces toxicity of polyglutamine expansions in fly and mouse models of Huntington disease. Nat Genet 36: 585-595, 2004

31. Schaefer CA, Kuhlmann CRW, Weiterer S, et al: Statins inhibit hypoxia-induced endothelial proliferation by preventing calcium-induced ROS formation. Atherosclerosis 185: 290-296, 2006.

32. Tuder RM, Groves B, Badesch DB and Voelkel NF: Exuberant endothelial cell growth and elements of inflammation are present in plexiform lesions of pulmonary hypertension. Am J Pathol 144: 275-285, 1994.

33. Yu L and Hales CA: Hypoxia does neither stimulate pulmonary artery endothelial cell proliferation in mice and rats with pulmonary hypertension and vascular remodeling nor in human pulmonary artery endothelial cells. J Vasc Res 48: 465-475, 2011.

34. Porter KM, Kang BY, Adesina SE, Murphy TC, Hart CM and Sutliff RL: Chronic hypoxia promotes pulmonary artery endothelial cell proliferation through $\mathrm{H}_{2} \mathrm{O}_{2}$-induced 5-lipoxygenase. PloS One 9: e98532, 2014.

35. Kang BY, Kleinhenz JM, Murphy TC and Hart CM: The PPAR $\gamma$ ligand rosiglitazone attenuates hypoxia-induced endothelin signaling in vitro and in vivo. Am J Physiol Lung Cell Mol Physiol 301: L881-L891, 2011.

36. Poon A, Eidelman D, Laprise C and Hamid Q: ATG5, autophagy and lung function in asthma. Autophagy 8: 694-695, 2012. 